\title{
Oncocytic Papillary Cystadenoma with Tyrosine-rich Crystalloids in the Parotid: Case Report with Cytology and Histology Correlation and Literature Review
}

\author{
Jamal Musayev ${ }^{1 *}$, Binnur Önal ${ }^{2}$, Chingiz Rahimov ${ }^{3}$, Adalat Hasanov ${ }^{1}$, Ismayil Farzaliyev ${ }^{3}$ and Mahira Nazirova ${ }^{4}$ \\ ${ }^{1}$ Department of Pathology, Azerbaijan Medical University, Baku, Azerbaijan \\ ${ }^{2}$ Department of Pathology and Cytology, Ankara Diskapı Teaching and Research Hospital, Ankara, Turkey \\ ${ }^{3}$ Department of Oral and Maxillofacial Surgery, Azerbaijan Medical University, Baku, Azerbaijan \\ ${ }^{4}$ Unification of Forensic Medical Expertise and Pathological Anatomy, Baku, Azerbaijan
}

"Corresponding author: Jamal Musayev, Department of Pathology, Azerbaijan Medical University, Baku, Azerbaijan, Tel: 00994503393684; E-mail: patolog.jamalmusaev@gmail.com

Received date: Jul 26, 2015, Accepted date: Aug 27, 2015; Published date: Aug 29, 2015

Copyright: (c) 2015 Musayev J, et al. This is an open-access article distributed under the terms of the Creative Commons Attribution License, which permits unrestricted use, distribution, and reproduction in any medium, provided the original author and source are credited.

\begin{abstract}
Oncocytic papillary cystadenomas are rare benign salivary gland neoplasms. Minor salivary glands are frequent localization for this neoplasm. We report a case of oncocytic papillary cystadenoma of the parotid gland, occurring in a 53-year-old male as a slowly growing, well defined cystic nodule of $1.5 \mathrm{~cm}$ in diameter. Fine needle aspiration (FNA) was performed by using 25-gauge needle by the (cyto) pathologist. Few small sheets of epithelial and oncocytic degenerative cells with extensive granular debris on the background were observed on FNA smears. Few tabular or petal form, non-birefringent crystalloids were noted in the background of scanty cellular specimens composed predominantly of oncocytic cells. On histopathology, unilocular cystic lesion with papillary structures lined by oncocytic cells and tyrosine-rich crystalloids within the lumen were identified. In conclusion, oncocytic papillary cystadenoma is uncommon benign tumor in the parotid gland and can be accompanied by tyrosine-rich crystalloids. It is useful to be familiar with the type of the crystalloid as they may help in differentiating benign and malignant lesions. To the best of authors' knowledge, this is the second reported case of oncocytic papillary cystadenoma with crystalloids with cyto-histo correlation.
\end{abstract}

Keywords: Papillary cystadenoma; Oncocytic cells; Parotid gland; Tyrosine-rich crystalloid; Fine needle aspiration cytology

\section{Introduction}

Cystadenoma is an uncommon benign epithelial neoplasm of salivary glands [1]. The majority of these tumors are located in minor salivary glands and about $45 \%$ of cases arise in the parotid gland [1]. Histologically, they are composed of cystic spaces lined by single layer of epithelial cells. Most cases are multilocular and 20\% of cystadenomas are unilocular. Extensive oncocytic metaplasia of epithelial layer in addition to the crystalloids within the cyst-lumen are rare; to the best of our knowledge, five cases of oncocytic papillary cystadenoma with crystalloids have been reported [2-6]. Fine needle aspiration (FNA) was applied to seven cases of several parotid lesions with crystalloids reported in the literature however only one previous case of oncocytic papillary cystadenoma with crystalloids has included FNA in diagnostic work-up [3,7-9]. We report a case of parotid oncocytic papillary cystadenoma with crystalloids with a review of the literature. To the best of our knowledge, the case presented here represents the second reported case of oncocytic papillary cystadenoma with crystalloids with cyto-histo correlation.

\section{Case report}

A 53-year-old male was admitted to the Department of Oral and Maxillofacial Surgery with a swelling in the right parotid region since 5 years associated with slight pain. Ultrasonography displayed a homogeneously hypoechoic lesion of size $1.5 \mathrm{~cm}$ with posterior acoustic enhancement, consistent with a benign cystic mass in the superficial lobe of the right parotid gland. By ultrasound and physical examination, the cervical lymph nodes were without any changes. FNA was performed by the (cyto) pathologist using 25-gauge needle and $20 \mathrm{~mL}$ syringe attached to the syringe-holder. Little amount of viscous-quality fluid was obtained by aspiration, not suitable for centrifugation or filtration. Two direct smears were prepared by the (cyto) pathologist; one smear was air-dried for May-Grünwald-Giemsa (MGG) staining while the other one was alcohol-fixed for Papanicolaou (PAP) method. No residual mass was detected to continue the aspiration. Few small sheets of epithelial and oncocytic degenerative cells, and foamy macrophages with extensive granular debris on the background were observed in hypocellular smears. Few tabular-form crystalloids with blunt ends, staining dark blue by MGG and faintly by PAP stainings were noted in the background of scanty cellular specimens composed predominantly of oncocytic cells and they were not discernible under polarized-light (Figure 1).

Cytologic material was reported as benign cytology, being compatible with "oncocytic cystic lesion of the salivary gland" and microscopic data concerning the crystalloids were noted. The patient underwent a right superficial parotidectomy. In gross examination, a cystic lesion measuring $15 \times 12 \times 7 \mathrm{~mm}$ was detected within the parotid gland. The external surface of the lesion was smooth and it was soft in consistency. On sectioning, the cut surface revealed an unilocular cyst with relatively smooth inner walls. The tissue was fixed in neutral buffered formalin and embedded in paraffin to be stained with hematoxylin and eosin (H\&E). 
Citation: Musayev J, Onal B, Rahimov C, Hasanov A, Farzaliyev I, et al. (2015) Oncocytic Papillary Cystadenoma with Tyrosine-rich Crystalloids in the Parotid: Case Report with Cytology and Histology Correlation and Literature Review. J Cytol Histol 6: 361 . doi:

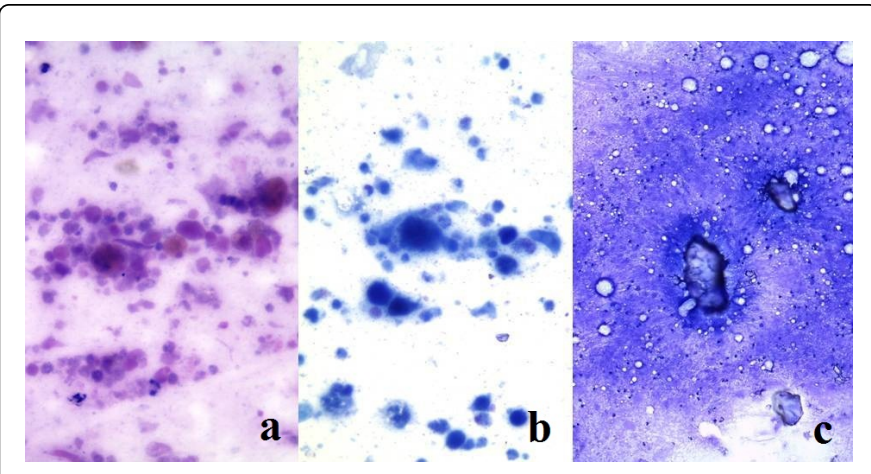

Figure 1: (a, b) Small sheets of epithelial and oncocytic degenerative cells with extensively granular background (c) few tabular/petal form with blunt ends and non-birefringent crystals on FNA smears (a: PAP $\times 100$; b and c: $M G G \times 100)$.

Subsequent histopathological examination showed unilocular cystic lesion with papillary structures lined by oncocytic cells and some crystalloids within the luminal space of the lesion. The microscopic and staining features of the crystalloids were evaluated as being compatible with tyrosine-rich crystalloids. There was a thick connective tissue layer partially surrounding the cystic cavity. The epithelial lining cells displayed the characteristic oncocytic features such as single round nucleus with conspicuous central nucleolus and abundant eosinophilic granular cytoplasm (Figure 2). The tabularform, non-birefringent crystalloids stained pale with a yellow hue on H\&E slides (Figure 3). No mitotic activity, cellular atypia or necrosis was encountered in either connective tissue or in the epithelial/ oncocytic component. Histomorphological diagnosis was rendered as "oncocytic papillary cystadenoma, displaying tyrosine-rich crystalloids". There were no complications or recurrence during 12month follow-up.

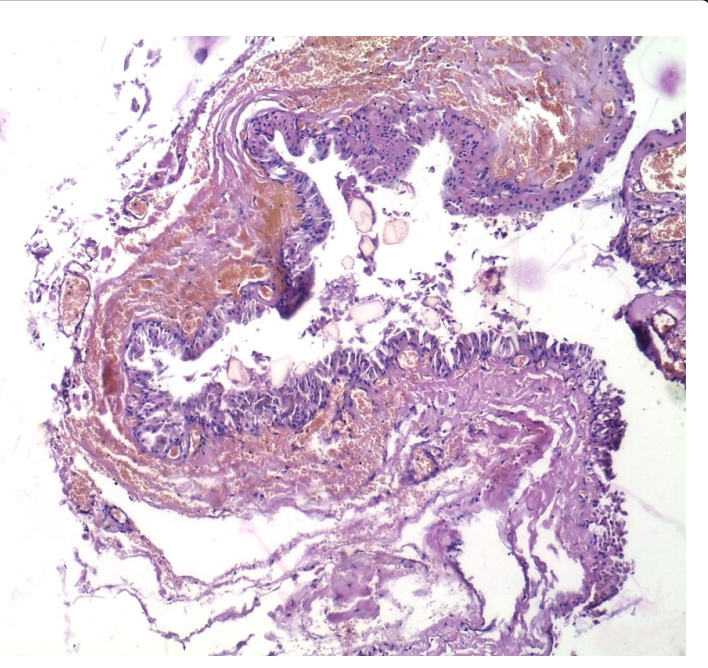

Figure 2: Unilocular cystic lesion with papillary structures lined by oncocytic cells $(\mathrm{H} \& \mathrm{E} \times 40)$.

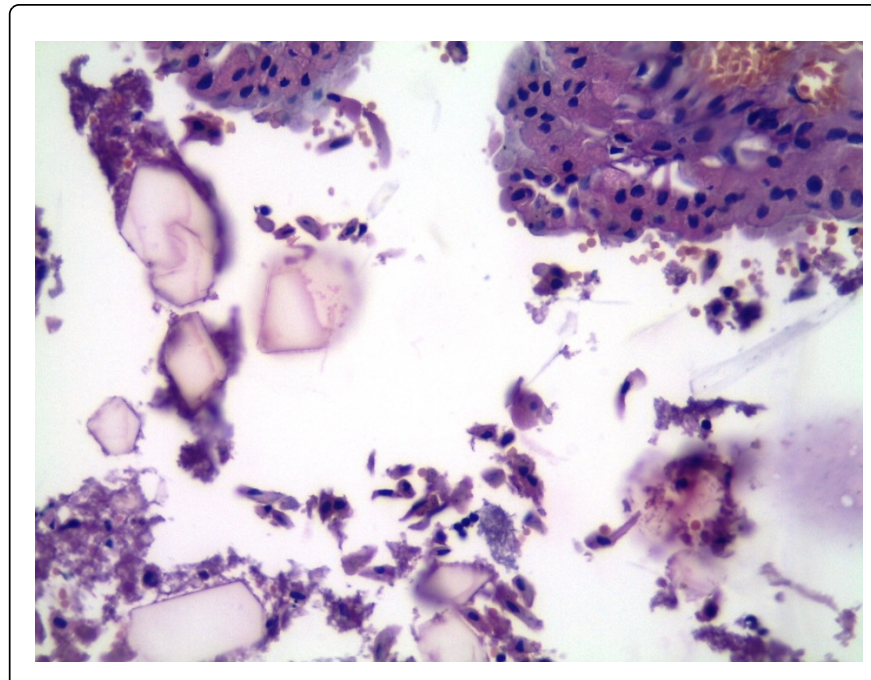

Figure 3: Tyrosine-rich crystals with tabular/petal shaped morphology with blunt ends, within the lumen of the cystic lesion $(\mathrm{H} \& \mathrm{E} \times 400)$.

\section{Discussion}

The frequency of cystadenoma is between $4.2-4.7 \%$ out of benign salivary gland tumours [1]. There is a female predominance and the mean age of the patients is about 57 years [1].

The oncocytic variant of cystadenoma is characterized by a single or double layer of oncocytes lining the cyst walls, resembling Warthin's tumor without the lymphoid stroma. Clinically, cystadenoma presents as a slow growing, painless cystic mass, usually smaller than $1 \mathrm{~cm}$ in diameter with the first impression of a mucocele. It can be detected by routine radiological methods easily and accordingly, sonography has been observed as the most applied method in diagnostic process of the reported cases.

Histopathologically, there are mucinous and papillary types of cystadenoma. Papillary cystadenoma are well-defined, unilocular or multilocular cysts, with intraluminal papillary projections $[7,10]$. The lining epithelial cells are cuboidal or columnar, and usually two layers in thickness. Oncocytic differentiation occurs rare and can be extensive [11]. Cases exhibiting with apocrine and focal squamous metaplasia and ciliated pseudostratified columnar epithelium have also been reported $[9,12,13]$.

FNA material of papillary cystadenoma is usually adequate for assessment. In the aspiration cytology; papillary structures, sheets of benign epithelial/oncocytic cells, histiocytes/macrophages with granular debris on the background could be seen [3,7-9]. In the literature, there was not any case of papillary cystadenoma reported as false positive by FNA. However it should always be kept in mind that any cellular elements may be diluted in a considerable specimen volume and this emphasizes the need for careful aspiration for cystic lesions and for repeating the aspiration for the residual masses of the salivary gland [14].

Most cases of salivary glands can be approached by FNA with growing confidence by clinicians and (cyto) pathologists. Several institutions indicate that the results obtained when (cyto) pathologists both perform and interpret aspirations are better than when these 
Citation: Musayev J, Onal B, Rahimov C, Hasanov A, Farzaliyev I, et al. (2015) Oncocytic Papillary Cystadenoma with Tyrosine-rich Crystalloids in the Parotid: Case Report with Cytology and Histology Correlation and Literature Review. J Cytol Histol 6: 361 . doi: $10.4172 / 2157-7099.1000361$

Page 3 of 4

functions are split between a clinician and a pathologist who has not seen the patient $[14,15]$.

There are quite a few cases of oncocytic papillary cystadenoma with intraluminal crystalloids reported in the English literature. Crystals in different shapes and nature may associate oncocytic type of salivary gland neoplasms. Only in 5 cases of cystadenoma; crystalloids $(2$ tyrosine-rich, 1 intraluminal and 2 non-tyrosine) have been reported; the location of the lesions were major salivary glands (4 lesions in parotid and 1 lesion in submandibular). Oncocytic metaplasia was seen in all of the cases with crystalloids [2-6].

Several types of crystalline structures such as amylase, tyrosine, collagenous, oxalate and intraluminal crystalloids have been illustrated in neoplastic and nonneoplastic lesions of salivary gland [5,6,15-17]. Alpha-amylase ( $\alpha$-amylase) crystalloids are the most frequently encountered, non-birefringent, rhomboid-shaped structures with pointed ends that stain deep blue by MGG, bright orange with PAP, and pink by H\&E. They range from $5 \mu \mathrm{m}$ to $500 \mu \mathrm{m}$ in size. Tyrosine rich crystalloids have petal-shaped or sun-burst morphology with blunt ends. Collagenous crystalloids are observed as radially-arranged needle-shaped fibers of collagen. Intraluminal crystalloids composed of dense amorphous eosinophilic material are identified in malignant salivary gland tumors $[5,15-17]$.

a-amylase crystalloids have so far been reported only in benign lesions while tyrosine-rich crystalloids occur mainly in pleomorphic adenomas and rarely in malignant salivary gland neoplasms. Collagenous crystalloids have been detected in pleomorphic adenomas and myoepitheliomas $[15,16]$.

Gilrease et al. [5] examined the prevalence of crystals and crystalloids in oncocytic salivary gland neoplasms. They detected tyrosine-rich crystals as non-birefringent and staining pink with $\mathrm{H} \& \mathrm{E}$, although the tabular forms also exhibiting a focal yellow hue similar to our case. They stained a red-brown color with Millon's reagent, which indicated the presence of tyrosine. Trichrome, periodic acid-Schiff stain with diastase, alcian blue ( $\mathrm{pH} 2.5)$, and Congo red stains were negative. Electron microscopy revealed sharply defined, elongated, electron-dense structures with periodicity, both extracellular and within epithelial cells. Their findings indicated that tyrosine-rich crystals associated with several oncocytic salivary gland neoplasms are morphologically, histochemically, and ultrastructurally distinct from previously described tyrosine-rich crystalloids of pleomorphic adenomas. Although the crystals appear to form by the assembly of small units within epithelial cells, the exact mode of formation remains unclear, according to them.

Nasuti et al. [3] proposed a relationship between non-tyrosine ( $\alpha$ amylase) crystalloids and cells with oncocytic features. They speculated that non-tyrosine crystalloids might represent a secretory product of salivary gland oncocytes since in all of their cases $(n=7)$, the nontyrosine crystalloids were found in highest concentrations in cystic spaces lined with oncocytic metaplastic cells.

On the other hand, Bezic et al. [6] focused on crystalloids arising in association with oncocytic cystadenomas specifically and displayed that the crystalloids in the majority of cases were of the tyrosine-rich type, as in our case. One possible explanation for this discrepancy is that some examples of non-tyrosine ( $\alpha$-amylase) crystalloids may have been erroneously reported as tyrosine-rich because they showed histochemical positivity for Millon's reagent, used to detect the presence of tyrosine. $\alpha$-amylase is a complex protein and contains some tyrosine and may therefore exhibit histochemical positivity for
Millon's reagent. In addition, the authors pointed out that the crystalloids associated with salivary oncocytic neoplasms, as reported in the study by Gilcrease et al. (five Warthin's tumors, one oncocytoma, and one oncocytic cystadenoma) were morphologically more similar to $\alpha$-amylase crystalloids than tyrosine-rich crystalloids.

Similarly, Chaushu et al. [18] clearly stated that in their case of multiple oncocytic cysts with crystalloids; microscopic and ultrastructural features of the crystalloids were compatible with $\alpha$ amylase crystalloids; however, the crystalloids were histochemically positive for tyrosine and non-immunoreactive for human salivary $a-$ amylase.

In accordance with the suggestion that intraluminal crystalloids in some reported cases of oncocytic cystadenomas are actually of the non-tyrosine type (instead of the tyrosine-rich type) were proven to be true, this could help confirm the possible relationship between nontyrosine crystalloids and oncocytic cells.

The differential diagnosis of oncocytic papillary cystadenoma should include benign and malignant salivary gland lesions consisting predominantly oncocytic cells and papillary components such as oncocytoma, Warthin's tumor (papillary cystadenoma lymphomatosum), pleomorphic adenoma with predominant oncocytic metaplasia, intraductal papilloma, low-grade mucoepidermoid carcinoma, the papillary-cystic variant of acinic cell carcinoma, polymorphous low-grade adenocarcinoma and cystadenocarcinoma $[1,12,15]$. Intraductal papilloma is an extremely rare benign tumor that arises most commonly in the minor salivary glands. They are cystic, solitary neoplasms that originate from ductal epithelium and produce painless swellings from which fluid is usually aspirated [15].

Cystadenomas are benign tumours, and conservative but complete surgical removal is recommended. Oncocytic papillary cystadenomas are unlikely to recur but rare cases of mucinous cystadenoma with malignant transformation have been described [1].

\section{Conclusion}

Oncocytic papillary cystadenoma is uncommon benign tumor in the parotid gland and can be accompanied by tyrosine-rich crystalloids. It is important to be aware of their existence and of stepwise diagnostic approach to salivary gland FNA since the cystic oncocytic and papillary lesions may pose difficulties in differential diagnosis. It is useful to be familiar with the type of crystalloid as they may help in differentiating benign and malignant lesions.

\section{References}

1. Skalova A, Michal M (2005) Cystadenoma. In: Barnes L, Eveson JW, Reichart P, Sidransky D (eds). Pathology and Genetics of Head and Neck Tumours. World Health Organisation, IARC Press, Lyon, pp 273-274.

2. Skálová A, Leivo I, Wolf H, Fakan F (2000) Oncocytic cystadenoma of the parotid gland with tyrosine-rich crystals. Pathol Res Pract 196: 849-851.

3. Nasuti JF, Gupta PK, Fleisher SR, LiVolsi VA (2000) Nontyrosine crystalloids in salivary gland lesions: report of seven cases with fineneedle aspiration cytology and follow-up surgical pathology. Diag Cytopathol 22: 167-171.

4. Başak K, Kiroğlu K (2014) Multiple oncocytic cystadenoma with intraluminal crystalloids in parotid gland: case report. Medicine (Baltimore) 93: e246. 
Citation: Musayev J, Onal B, Rahimov C, Hasanov A, Farzaliyev I, et al. (2015) Oncocytic Papillary Cystadenoma with Tyrosine-rich Crystalloids in the Parotid: Case Report with Cytology and Histology Correlation and Literature Review. J Cytol Histol 6: 361 . doi: $10.4172 / 2157-7099.1000361$

Page 4 of 4

5. Gilcrease MZ, Nelson FS, Guzman-Paz M (1998) Tyrosine-rich crystals associated with oncocytic salivary gland neoplasms. Arch Pathol Lab Med 122: 644-649.

6. Bezić J, Glavina-Durdov M (2011) Salivary oncocytic cystadenoma with intraluminal crystalloids: A case report with review of the literature. Head Neck Pathol 5: 419-422.

7. Lim CS, Ngu I, Collins AP, McKellar GM (2008) Papillary cystadenoma of a minor salivary gland: Report of a case involving cytological analysis and review of the literature. Oral Surg Oral Med Oral Pathol Oral Radiol Endod 105: e28-33.

8. Chin S, Kim HK, Kwak JJ (2014) Oncocytic papillary cystadenoma of major salivary glands: Three rare cases with diverse cytologic features. J Cytol 31: 221-223.

9. Zhang S, Bao R, Abreo F (2009) Papillary oncocytic cystadenoma of the parotid glands: A report of 2 cases with varied cytologic features. Acta Cytol 53: 445-448.

10. Gallego L, Junquera L, Fresno MF, de Vicente JC (2008) Papillary cystadenoma and cystadenocarcinoma of salivary glands: two unusual entities. Med Oral Patol Oral Cir Bucal 13: E460-463.

11. Ellis GL, Auclair PL (2008) Tumors of the salivary glands: AFIP Atlas of Tumor Pathology. (4thedn.) Silver Spring MD: ARP Press, Washington, pp 225-230.
12. Stathopoulos P, Gagari E (2013) Papillary cystadenoma of the lower lip exhibiting ciliated pseudostratified columnar epithelium: report of a bizarre case and review of the literature. Oral Maxillofac Surg 17: 161-164.

13. Halbritter SA, Altermatt HJ, Caversaccio M, Bornstein MM (2009) Apocrine papillary cystadenoma of a minor salivary gland on the lower lip: case presentation. Quintessence Int 40: 167-169.

14. Önal B (2001) Who should perform aspiration?: Role of cytopathology and function of fine needle aspiration unit in cancer diagnosis and management. Health Ministry of Turkey publications, Ankara, pp 66-69.

15. Kocjan G (2001) Salivary glands: Clinical cytopathology of the head and neck. Greenwich Medical Media, London.

16. Issac RM, Oommen AM, Mathai JM (2014) Crystalloids in salivary gland lesions. J Cytol 31: 194-195.

17. Pantanowitz L, Goulart RA, Jackie Cao Q (2006) Salivary gland crystalloids. Diagn Cytopathol 34: 749-750.

18. Chaushu G, Buchner A, David R (1999) Multiple oncocytic cysts with tyrosine-crystalloids in the parotid gland. Hum Pathol 30: 237-239. 